\title{
INVARIANT MEASURES IN FREE MV-ALGEBRAS
}

\author{
GIOVANNI PANTI
}

\begin{abstract}
MV-algebras can be viewed either as the Lindenbaum algebras of Łukasiewicz infinite-valued logic, or as unit intervals of lattice-ordered abelian groups in which a strong order unit has been fixed. The free $n$-generated MValgebra Free $_{n}$ is representable as an algebra of continuous piecewise-linear functions with integer coefficients over the unit cube $[0,1]^{n}$. The maximal spectrum of Free $_{n}$ is canonically homeomorphic to $[0,1]^{n}$, and the automorphisms of the algebra are in 1-1 correspondence with the pwl homeomorphisms with integer coefficients of the unit cube. In this paper we prove that the only probability measure on $[0,1]^{n}$ which is null on underdimensioned 0 -sets and is invariant under the group of all such homeomorphisms is the Lebesgue measure. From the viewpoint of lattice-ordered abelian groups, this fact means that, in relevant cases, fixing an automorphism-invariant strong unit implies fixing a distinguished probability measure on the maximal spectrum. From the viewpoint of algebraic logic, it means that the only automorphism-invariant truth averaging process that detects pseudotrue propositions is the integral with respect to Lebesgue measure.
\end{abstract}

\section{Preliminaries}

An $M V$-algebra is an algebra $(A, \oplus, \neg, 0)$ such that $(A, \oplus, 0)$ is a commutative monoid and the identities $\neg \neg f=f, f \oplus \neg 0=\neg 0$, and $\neg(\neg f \oplus g) \oplus g=\neg(\neg g \oplus$ $f) \oplus f$ are satisfied. MV-algebras can be viewed either as the Lindenbaum algebras of Łukasiewicz infinite-valued logic, or as unit intervals of lattice-ordered abelian groups ( $\ell$-groups) in which a strong order unit has been fixed. See [2, 1], 4] for $\ell$-groups, and [6], 8], 3] for MV-algebras and Lukasiewicz logic.

We recall that a strong unit in an $\ell$-group $G$ is a positive element $u$ of $G$ such that for every $g \in G$ there exists a positive integer $n$ for which $g \leq n u$. The unit interval $\Gamma(G, u)=\{g \in G: 0 \leq g \leq u\}$ is then an MV-algebra under the operations $f \oplus g=(f+g) \wedge u, \neg f=u-f, 0=0_{G}$, and the functor $\Gamma$ is an equivalence between the category of $\ell$-groups with a distinguished strong unit and the category of MValgebras [6]. A state on $(G, u)$ is a group homomorphism $m: G \rightarrow \mathbb{R}$ which is positive $(f \geq 0$ implies $m(f) \geq 0)$ and normalized $(m(u)=1)$ [5, Chapter 4]. The same definition, recast in terms of MV-algebras, amounts to the following: a state on the MV-algebra $A$ is a function $m: A \rightarrow[0,1]$ such that $m(0)=0, m(\neg 0)=1$, and $m(f \oplus g)=m(f)+m(g)$ provided that $\neg(\neg f \oplus \neg g)=0$ []. If $A$ is viewed as the Lindenbaum algebra of some theory in Eukasiewicz logic, then $m$ is a function assigning an "average truth-value" to the elements of $A$, i.e., to the propositional formulas modulo the theory.

Key words and phrases. MV-algebras, state, automorphism-invariance, piecewise-linear homeomorphisms.

2000 Math. Subj. Class.: 06D35; 37A05. 
The $\Gamma$ functor induces a canonical bijection between the states of $(G, u)$ and those of $A=\Gamma(G, u)$ and a homeomorphism between the maximal spectrum of $(G, u)$ and $\operatorname{MaxSpec} A$. We recall that the maximal spectrum of $(G, u)$ is the set of maximal $\ell$-ideals of $G$, while MaxSpec $A$ is the set of maximal ideals of $A$ (i.e., kernels of homomorphisms from $A$ to $\Gamma(\mathbb{R}, 1)$ ); both sets are equipped with the Zariski topology [2, Chapitre 10]. We will formulate our results mainly in terms of $\mathrm{MV}$-algebras, leaving to the reader their straightforward translation in the language of $\ell$-groups with strong unit.

The set of all states of $A$ is a compact convex subset of $[0,1]^{A}$, where the latter is given the product topology, and the subspace $X$ of extremal states (a state $m$ is extremal if it cannot be expressed as $m=t p+(1-t) q$, with $p, q$ distinct states and $0<t<1$ ) is homeomorphic to MaxSpec $A$. This homeomorphism is canonical: if $\mathfrak{p}$ is a maximal ideal, then there is a unique embedding of $\mathrm{MV}$ algebras $A / \mathfrak{p} \rightarrow \Gamma(\mathbb{R}, 1)=[0,1]$, and the extremal state $p$ corresponding to $\mathfrak{p}$ is the composition of this embedding with the quotient map $A \rightarrow A / \mathfrak{p}$. We identify $X$ with $\operatorname{MaxSpec} A$, and we write $f(p)$ for the image of $f / \mathfrak{p}$ in $[0,1]$. As a function, $f$ is an element of $C(X)$ (the Banach vector lattice of real-valued continuous functions on $X$ endowed with the uniform norm \|\|$\left._{\infty}\right)$, and the resulting map $A \rightarrow \Gamma(C(X), 1)$ (here 1 is the characteristic function of $X$ ) is a homomorphism of MV-algebras whose kernel is the radical ideal $\mathfrak{r}=\bigcap \operatorname{MaxSpec} A$ of $A$.

Proposition 1.1. Let $A$ be an $M V$-algebra, $X=\operatorname{MaxSpec} A, P(X)$ the set of all regular Borel probability measures on $X$. Then the states on $A$ are in 1-1 correspondence with the elements of $P(X)$.

Proof. The states of $A$ are in 1-1 correspondence with the states of $A / \mathfrak{r}[\underline{8}$, Proposition 3.1(3)]. We may then assume that $A$ has 0 radical, and identify $A$ with a separating subalgebra of $\Gamma(C(X), 1)$. Obviously every Borel probability measure $\mu$ on $X$ induces a state $m$ on $A$ via Lebesgue integration:

$$
m(f)=\int_{X} f d \mu .
$$

We must show that the map $\mu \mapsto m$, when restricted to the set of regular measures, is a bijection. Let then $m$ be a state on $A$, which uniquely corresponds to a state - again denoted by $m$ - on the $\ell$-group with strong unit $(G, 1) \subseteq(C(X), 1)$ enveloping $A$ [8, Theorem 2.4]. Since $G$ is torsion-free, it embeds in its divisible hull $H=\{q f: q \in \mathbb{Q}, f \in G\}$, which is a sub-vector lattice of $C(X)$ over the rationals. One sees easily that $m$ extends uniquely to a state on $(H, 1)$, via $m(q f)=q m(f)$. By the lattice version of the Stone-Weierstrass Theorem, $H$ is dense in $C(X)$. Since $m$ is positive, it is bounded $\left(\|f\|_{\infty} \leq 1 \Longrightarrow-1 \leq f \leq 1 \Longrightarrow\right.$ $m(-1) \leq m(f) \leq m(1) \Longrightarrow|m(f)| \leq m(1))$, whence continuous. Therefore $m$ can be extended uniquely to a state on $(C(X), 1)$ via $m(f)=\lim m\left(h_{n}\right)$, whenever $f \in C(X)$ is the uniform limit of elements $h_{n} \in H$. Note that $m$ is still positive on $C(X)$, since every $0 \leq f \in C(X)$ can be written as the limit of positive elements of $H$. The proof is now completed by an application of the Riesz Representation Theorem [15, Theorem 2.14], which guarantees the existence of a unique $\mu \in P(X)$ that induces $m$.

Let us note that if every open set in $X$ is $\sigma$-compact (i.e., a countable union of compact sets), then every Borel measure on $X$ is regular [15, Theorem 2,18]. This applies, in particular, when $A$ is a countable MV-algebra. Indeed, in this case $X$ is 
second countable, whence is metrizable and has $\sigma$-compact open sets. For countable $A$, we can then remove the word "regular" in the statement of Proposition 1.1

If $A, B$ are $\mathrm{MV}$-algebras (not necessarily having 0 radical), $X=\operatorname{MaxSpec} A$, $Y=\operatorname{MaxSpec} B, \sigma: A \rightarrow B$ a homomorphism, then the dual $S: Y \rightarrow X$ of $\sigma$ is defined by $S(m)=m \circ \sigma$ (at the level of extremal states), or by $S(\mathfrak{p})=\sigma^{-1}[\mathfrak{p}]$ (at the level of maximal ideals). Recall that if $S: Y \rightarrow X$ is a Borel measurable map between topological spaces and $\mu$ is a Borel measure on $Y$, then the push-forward of $\mu$ by $S$ is the Borel measure $S_{*} \mu$ on $X$ defined by $\left(S_{*} \mu\right)(A)=\mu\left(S^{-1} A\right)$. If now $\mu \in P(Y)$ corresponds to $m$ as in Proposition 1.1, then $S(m)$ corresponds to $S_{*} \mu$. Indeed, for every $f \in A$, we have $\sigma(f)=f \circ S$ as a function on $Y$, and therefore

$$
[S(m)](f)=m(\sigma(f))=\int_{Y} \sigma(f) d \mu=\int_{Y} f \circ S d \mu=\int_{X} f d\left(S_{*} \mu\right) .
$$

If in particular $\sigma$ is an endomorphism of $A=B$, then $m$ is $\sigma$-invariant iff $(X, \mu, S)$ is a measure-theoretic dynamical system.

\section{The main Result}

Fix $n \geq 1$, and let Free ${ }_{n}$ be the free MV-algebra over $n$ generators. It is well known that Free ${ }_{n}$ has 0 radical and that $X=$ MaxSpec Free $_{n}$ is homeomorphic to the $n$-cube $[0,1]^{n}$; see [6, 7], 9] for all unproved claims on free MV-algebras. A rational polyhedral complex is a finite set $W$ of compact convex polyhedrons such that: (1) every polyhedron is contained in $[0,1]^{n}$, and all its vertices have rational coordinates; (2) if $C \in W$ and $D$ is a face of $C$, then $D \in W$; (3) every two polyhedra intersect in a common face. We write $|W|$ for the union of all elements of $W$. The range of the embedding Free ${ }_{n} \rightarrow \Gamma(C(X), 1)$ described before Proposition 1.1 is then the set of all McNaughton functions on $[0,1]^{n}$, i.e., those continuous functions $f:[0,1]^{n} \rightarrow[0,1]$ for which there exists a complex $W$ as above and affine linear functions $F_{j}(\bar{x})=a_{j 1} x_{1}+\cdots+a_{j n} x_{n}+a_{j(n+1)} \in \mathbb{Z}\left[x_{1}, \ldots, x_{n}\right]$, in 1-1 correspondence with the $n$-dimensional polyhedra $C_{j}$ of the complex, such that $|W|=[0,1]^{n}$ and $f\left\lceil C_{j}=F_{j}\right.$ for each $j$. The $i$ th projection $x_{i}:[0,1]^{n} \rightarrow[0,1]$ is the $i$ th free generator of Free $_{n}$. If $\sigma$ is an automorphism of Free ${ }_{n}$ then, upon the identification of MaxSpec Free ${ }_{n}$ with $[0,1]^{n}$, its dual is the homeomorphism $S:[0,1]^{n} \rightarrow[0,1]^{n}$ defined by $S(p)_{i}=\left(\sigma\left(x_{i}\right)\right)(p)$. This is equivalent to saying that there exists a complex $W$ as above and matrices $P_{j} \in \operatorname{Mat}_{n+1}(\mathbb{Z})$, in 1-1 correspondence with the $n$-dimensional polyhedra $C_{j}$ of $W$, such that $|W|=[0,1]^{n}$ and:

(1) every $P_{j}$ has last row $(0 \ldots 01)$;

(2) $P_{j}$ expresses $S\left\lceil C_{j}\right.$ in homogeneous coordinates (i.e., if $p=\left(r_{1}, \ldots, r_{n}\right) \in$ $C_{j}$ and $S(p)=\left(s_{1}, \ldots, s_{n}\right)$, then $\left.\left(s_{1} \ldots s_{n} 1\right)^{t r}=P_{j}\left(r_{1} \ldots r_{n} 1\right)^{t r}\right)$;

(3) all $P_{j}$ have determinant +1 , or they all have determinant -1 .

We call these duals McNaughton homeomorphisms of the unit cube. Note that each of $\sigma$ and $S$ determines the other; indeed, given $S$ as above, $\sigma$ is the automorphism that maps $x_{i}$ to $x_{i} \circ S$.

Throughout this paper $\lambda$ (or $\lambda^{n}$, when clarity requires) will denote the Lebesgue measure on $[0,1]^{n}$. We simplify notation by denoting a Borel measure and the state it induces by the same Greek letter. As remarked in [8], $\lambda$ has the following properties:

(A) $\lambda(f) \in \mathbb{Q}$ for every $f \in$ Free $_{n}$; 
(B) it is faithful: $f \neq 0$ implies $\lambda(f) \neq 0$;

(C) it is automorphism-invariant: for every $f$ and every automorphism $\sigma$ of Free $_{n}$, we have $\lambda(\sigma(f))=\lambda(f)$ (equivalently, for every Borel $A \subseteq[0,1]^{n}$ and every McNaughton homeomorphism $S$, we have $\lambda(A)=\lambda\left(S^{-1} A\right)$ ).

These properties do not characterize $\lambda$. Indeed, define the denominator of the rational point $p=\left(r_{1}, \ldots, r_{n}\right) \in[0,1]^{n} \cap \mathbb{Q}^{n}$ as the unique integer $d=\operatorname{den}(p) \geq 1$ such that $d r_{1}, \ldots, d r_{n}, d$ are relatively prime integers. For a fixed $d$, the set $A_{d}^{n}$ of all points in $[0,1]^{n}$ having denominator $d$ has finite cardinality $\# A_{d}^{n}$, and every McNaughton homeomorphism permutes $A_{d}^{n}$.

Example 2.1. Choose $d \geq 1$, and let $\nu_{d}^{n}$ be the counting measure on $A_{d}^{n}$, giving each point mass $1 / \# A_{d}^{n}$. Define

$$
\mu^{n}=\frac{1}{2} \lambda^{n}+\frac{1}{2} \nu_{d}^{n} .
$$

Then $\mu^{n}$ satisfies (A), (B), (C). These measures may exhibit pathological behaviour. Take, e.g., $d=4$ and $f\left(x_{1}\right)=\neg\left(\neg x_{1} \oplus \neg x_{1}\right)$. We have $A_{4}^{1}=\{1 / 4,3 / 4\}$, and direct computation shows that integration w.r.t. $\mu^{1}$ assigns $f$ value $1 / 4$. But we may as well consider $f$ as an element of Free 2 , possibly making explicit the dummy variable as, say, $f\left(x_{1}, x_{2}\right)=\neg\left(\neg x_{1} \oplus \neg x_{1}\right) \wedge\left(x_{2} \oplus \neg x_{2}\right)$. Then $\# A_{4}^{2}=16$, and $\mu^{2}(f)=17 / 64$. The trouble here is that $A_{4}^{2}$ does not map onto $A_{4}^{1}$ under the canonical projection.

Beyond showing that (A), (B), (C) do not characterize $\lambda$, Example 2.1 makes clear that we cannot assign measures to the various $n$-cubes in an unrelated way, and explains the need for the following condition:

(D) for each $n \geq 1$, let $\mu^{n}$ be a Borel probability measure on $[0,1]^{n}$. Let $\pi:[0,1]^{n+1} \rightarrow[0,1]^{n}$ be the canonical projection on the first $n$ coordinates. We say that the system $\left\{\mu^{n}\right\}$ is coherent if $\mu^{n}$ and the push-forward measure $\pi_{*} \mu^{n+1}$ coincide for every $n$.

We now state a condition that will characterize the system of Lebesgue measures.

Definition 2.2. As usual in lattice theory, the pseudocomplement of $f \in$ Free $_{n}$ is the largest $g$ (if it exists) such that $f \wedge g=0$. We say that $f$ is pseudotrue if it has pseudocomplement 0 . Let $\mu$ be a Borel measure on $[0,1]^{n}$, and write $k f$ for $f \oplus f \oplus \cdots \oplus f$ ( $k$ times). We say that $\mu$ detects pseudotruths if the following holds:

(E) for every pseudotrue $f, \sup \{\mu(k f): k \geq 1\}=1$.

The 0 -set of $f \in$ Free $_{n}$ is $Z f=\left\{p \in[0,1]^{n}: f(p)=0\right\}$. We have that $Z f=$ $|W|$ for some rational polyhedral complex $W$, and conversely any such complex is the 0 -set of some $f$; this follows from the theory of Schauder hats [7, [9]. The sequence $f, 2 f, 3 f, \ldots$ is nondecreasing, and its pointwise limit is the characteristic function of the support of $f$, the latter being supp $f=[0,1]^{n} \backslash Z f$. For every Borel measure $\mu$ on $[0,1]^{n}$, we have $\sup \mu(k f)=\lim \mu(k f)=\mu(\operatorname{supp} f)$ by the Monotone Convergence Theorem. For every nonempty open set $U$ there exists a $g \neq 0$ such that supp $g \subseteq U$ [6, Proposition 4.17]. Therefore, $f$ is pseudotrue iff $f \wedge g \neq 0$ for every $g \neq 0$ iff supp $g \nsubseteq Z Z f$ for every $g \neq 0$ iff $Z f$ has empty topological interior. Summing up, (E) is equivalent to

$\left(\mathrm{E}^{\prime}\right)$ if $W$ is a rational polyhedral complex such that $|W|$ has empty interior, then $\mu(|W|)=0$.

The following is our main result. 
Theorem 2.3. For every $n \geq 1$, let $\mu^{n}$ be an automorphism-invariant Borel measure on $[0,1]^{n}$ that detects pseudotruths. Then, for every $n \geq 2$, we have $\mu^{n}=\lambda^{n}$. If moreover the system $\left\{\mu^{n}\right\}$ is coherent, then $\mu^{1}=\lambda^{1}$ as well.

Let us look at Theorem 2.3 from the viewpoint of $\ell$-groups. Let $P=\{p \in$ $\left.\mathbb{R}^{n}: p_{1}, \ldots, p_{n} \geq 0\right\}$ be the positive cone of $\mathbb{R}^{n}$, and let $G_{n}$ be the $\ell$-group of all homogeneous pwl functions with integer coefficients from $P$ to $\mathbb{R}$, with pointwise operations. It is well known that $G_{n}$ is a projective $\ell$-group, and all finitely generated projective $\ell$-groups have such a form, i.e., are groups of homogeneous pwl functions with integer coefficients defined over some rational cone complex. The map $p \mapsto\left\{f \in G_{n}: f(p)=0\right\}$ is a homeomorphism between the $(n-1)$-dimensional simplex $\Delta^{n-1}=\left\{\sum \alpha_{i} e_{i}: \alpha_{i} \geq 0\right.$ and $\left.\sum \alpha_{i}=1\right\}$ (the $e_{i}$ 's being the standard basis vectors of $\mathbb{R}^{n}$ ) and the maximal spectrum of $G_{n}$. Now, $G_{n}$ has plenty of automorphisms, and there is no reason for the existence of a nontrivial probability measure on $\Delta^{n-1}$ invariant under [the duals of] all such automorphisms. The following simple example may clarify this situation.

Example 2.4. Let $n=2$, and let

$$
\begin{array}{ll}
p_{1}=e_{1}+e_{2}, & p_{2}=e_{1}+2 e_{2}, \\
p_{1}^{\prime}=2 e_{1}+e_{2}, & p_{2}^{\prime}=e_{1}+e_{2} .
\end{array}
$$

Let $s: P \rightarrow P$ be the homogeneous pwl map that fixes $e_{1}$ and $e_{2}$, maps $p_{i}$ to $p_{i}^{\prime}$, and is linear on each of the three cones $\mathbb{R}^{+} e_{1}+\mathbb{R}^{+} p_{1}, \mathbb{R}^{+} p_{1}+\mathbb{R}^{+} p_{2}, \mathbb{R}^{+} p_{2}+\mathbb{R}^{+} e_{2}$. Then $s$ is a homeomorphism of $P$, and the linear maps on each of the three cones above have integer coefficients. Therefore $s$ is induced by a unique automorphism $\sigma$ of $G_{2}$, which is explicitly given by

$$
\sigma\left(x_{1}\right)=x_{1} \vee\left[\left(3 x_{1}-x_{2}\right) \wedge\left(x_{1}+x_{2}\right)\right], \quad \sigma\left(x_{2}\right)=\left(-x_{1}+x_{2}\right) \vee\left[x_{1} \wedge x_{2}\right] .
$$

Parametrizing $\Delta^{1}$ via $[0,1] \ni t \mapsto(1-t) e_{1}+t e_{2}$, one sees that the dual $S$ of $\sigma$ is piecewise-fractional, namely

$$
S(t)= \begin{cases}t /(1+t), & \text { if } 0 \leq t<1 / 2 \\ (1-t) /(4-5 t), & \text { if } 1 / 2 \leq t<2 / 3 \\ (2 t-1) / t, & \text { if } 2 / 3 \leq t \leq 1\end{cases}
$$

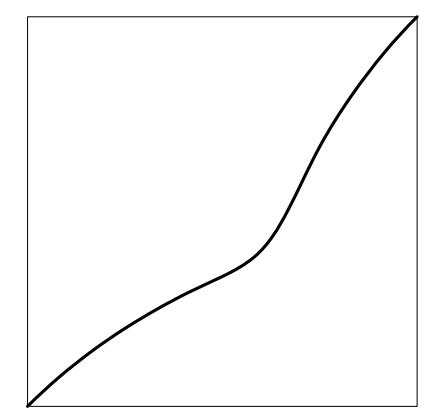

All $t \in[0,1]$, except the repelling fixed point 1 , is attracted under $S$ to the point 0 . This implies that the only $S$-invariant probability measures on $[0,1] \simeq \Delta^{1}$ 
are the convex combinations of the Dirac measures at 0 and 1 , a definitely trivial situation.

Things become more interesting if we fix a strong unit $u$ in $G_{n}$, and restrict attention to those automorphisms of $G_{n}$ that fix $u$. This amounts to studying the MV-algebra $\Gamma\left(G_{n}, u\right)$ and its group of automorphisms; the intuition behind is that fixing a strong unit in an $\ell$-group amounts to fixing a "scale of magnitude". This intuition is now supported by our Theorem 2.3. Indeed, a clever choice of $u$ makes $\Gamma\left(G_{n}, u\right)$ isomorphic to Free ${ }_{n-1}$ : this fact is proved in [10, Theorem 4.1], and we leave as an exercise for the reader to show that we can take $u=\left(x_{1} \vee x_{2} \vee \cdots \vee\right.$ $\left.x_{n-1}\right)+x_{n}$. Then the Lebesgue measure $\lambda$ on the maximal spectrum $\Delta^{n-1}$ of $G_{n}$ is invariant under [the duals of] all automorphisms of $G_{n}$ that leave $u$ fixed, and Theorem 2.3 says that this property, together with being null on underdimensioned 0 -sets, does indeed characterize $\lambda$.

Finally, the relevance of Theorem 2.3 from the viewpoint of algebraic logic is clear: it gives a distinguished status to the Lebesgue measure as an averaging measure on the space $[0,1]^{n}$ of truth-value assignments in Eukasiewicz infinite-valued logic. We only stress here that, although the proof of Theorem 2.3 exploits a fair amount of the arithmetic and piecewise-linear structure of $[0,1]^{n}$, the resulting characterization of the state on Free ${ }_{n}$ induced by integration w.r.t. the Lebesgue measure as the only automorphism-invariant state detecting pseudotruths is intrinsic and purely algebraic. We refer to [11, §2] for a detailed discussion on this issue.

\section{THE CASE $n=2$}

The key point in establishing Theorem 2.3 is the case $n=2$.

Theorem 3.1. Let $\mu$ be a Borel measure on $[0,1]^{2}$. Assume that $\mu$ detects pseudotruths and is automorphism-invariant. Then $\mu=\lambda$.

The analogous statement is false for $n=1$. Indeed, it is easy to show that the only McNaughton homeomorphisms of $[0,1]$ are the identity and the flip $p \mapsto$ $1-p$. Therefore, any flip-invariant strictly positive density $s$ (i.e., a $\lambda$-integrable function $s:[0,1] \rightarrow(0, \infty)$ such that $\int s d \lambda=1$ and $\left.s(p)=s(1-p)\right)$ determines a measure absolutely continuous w.r.t. $\lambda$, automorphism-invariant, faithful, and detecting pseudotruths. The trouble here is that $[0,1]$ has so few McNaughton homeomorphisms that rigidity phenomena cannot occur.

Rigidity is a recurrent theme in measurable dynamics. If $X$ is a space on which a transformation $R$ acts, then typically there is a multitude of $R$-invariant probability measures on $X$. But if we add a second transformation $S$ which is incommensurable with $R$ (i.e., no power of $R$ is a power of $S$ ), then the set of measures invariant for both $R$ and $S$ may shrink down drastically, often just to the "obvious" ones. The most famous, and still unsolved, problem in this area is the Furstenberg Conjecture: the only nonatomic probability on the unit circle $\{z \in \mathbb{C}:|z|=1\}$ which is invariant under both $z \mapsto z^{2}$ and $z \mapsto z^{3}$ is the Lebesgue measure.

We shall prove Theorem 3.1 using such a rigidity approach. I must sincerely thank Carlangelo Liverani and François Ledrappier for explaining me the rigidity of the two-dimensional torus under the action of $\mathrm{SL}_{2} \mathbb{Z}$; the insight they gave was the starting point of the present work. 
We will denote rational points of the unit square switching freely between nonhomogeneous and homogeneous coordinates, e.g., the center of the square is either $(1 / 2,1 / 2)$ or $(1: 1: 2)$. For $h \geq 0$, define $p_{0}^{h}=(h+1: h: 2 h+1)$, $p_{1}^{h}=(h+1: h+1: 2 h+1), p_{2}^{h}=(h: h+1: 2 h+1), p_{3}^{h}=(h: h: 2 h+1)$. For every $k \geq 0$, we define a McNaughton homeomorphism $R_{k}$ by considering the following rational polyhedral complex (we draw the picture for $k=1$ ):

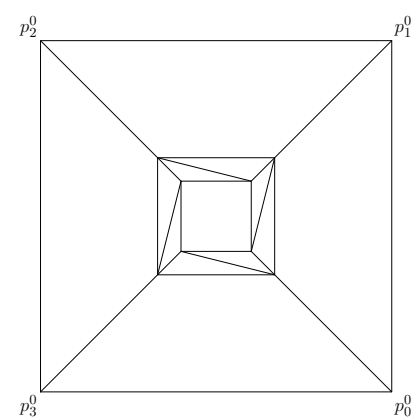

The vertices of the intermediate square are $p_{i}^{k}$, for $i=0, \ldots, 3$, and those of the inner square are $p_{i}^{k+1}$. Let $R_{k}^{\prime}$ be the unique homeomorphism that:

(1) $\operatorname{maps} p_{i}^{k+1}$ to $p_{i+1(\bmod 4)}^{k+1}$;

(2) fixes every other vertex;

(3) is affine linear on each polyhedron.

By direct computation, one sees that $R_{k}^{\prime}$ is a McNaughton homeomorphism. E.g., if $T$ is the triangle $\left\langle p_{0}^{k+1}, p_{0}^{k}, p_{1}^{k}\right\rangle$, then $R_{k}^{\prime}[T]$ is the triangle $\left\langle p_{1}^{k+1}, p_{0}^{k}, p_{1}^{k}\right\rangle$, and the matrix expressing $R_{k}^{\prime} \uparrow T$ in homogeneous coordinates is

$$
\left(\begin{array}{ccc}
1 & 0 & 0 \\
-2 k-1 & 1 & k+1 \\
0 & 0 & 1
\end{array}\right)
$$

Define $R_{k}=\left(R_{k}^{\prime}\right)^{4}$ to be the fourth power of $R_{k}^{\prime}$.

Let now $D$ be the unit disk in polar coordinates $(r, \theta)$, for $-\pi<\theta \leq \pi$, and let $M: D \rightarrow[0,1]^{2}$ be the homeomorphism defined by:

$$
M(r, \theta)=(1 / 2,1 / 2)+ \begin{cases}r(-1 / 2,-2 \theta / \pi-3 / 2), & \text { if }-\pi<\theta \leq-\pi / 2 ; \\ r(2 \theta / \pi+1 / 2,-1 / 2), & \text { if }-\pi / 2<\theta \leq 0 ; \\ r(1 / 2,2 \theta / \pi-1 / 2), & \text { if } 0<\theta \leq \pi / 2 ; \\ r(-2 \theta / \pi+3 / 2,1 / 2), & \text { if } \pi / 2<\theta \leq \pi .\end{cases}
$$

For every $r \in[0,1]$, let $K_{r}=\{(r, \theta):-\pi<\theta \leq \pi\}$ be the circle of radius $r$, let $Q_{r}=M\left[K_{r}\right]$ (it is the boundary of a square centered in $(1 / 2,1 / 2)$ ), and let $t_{k}:[0,1] \rightarrow[0,1]$ be the continuous piecewise-fractional function defined by

$$
t_{k}(r)=\min (1, \max (0,1 /(2 r)-k-1 / 2)) .
$$

Lemma 3.2. The homeomorphism $M$ provides a conjugation between $R_{k}$ and the map $T_{k}: D \rightarrow D$ given by $T_{k}(r, \theta)=\left(r, \theta+2 \pi t_{k}(r)\right)$ (i.e., $\left.R_{k} \circ M=M \circ T_{k}\right)$.

Proof. Let $v \in K_{r}$. We may assume $1 /(2(k+1)+1)<r<1 /(2 k+1)$, since otherwise $T_{k}$ fixes $v$ and $R_{k}$ fixes $M(v)$. If $u$ is another point in $K_{r}$, we define the oriented 
distance between $u$ and $v$ as the length $d(u, v)$ of the path which goes from $u$ to $v$ along $K_{r}$ in the counterclockwise direction (we normalize the total length of $K_{r}$ to be 1). We define analogously the oriented distance of two points of $Q_{r}$. The differential of $M$ maps the unit tangent vector $\partial /\left.\partial \theta\right|_{u}$ to $K_{r}$ at $u$ to a vector of constant modulus, namely either $-2 r / \pi \partial /\left.\partial y\right|_{M u}$, or $2 r / \pi \partial /\left.\partial x\right|_{M u}$, or $2 r / \pi \partial /\left.\partial y\right|_{M u}$, or $-2 r / \pi \partial /\left.\partial x\right|_{M u}$, depending on $\theta \in(-\pi, \pi) \backslash\{0, \pm \pi / 2\}$. Therefore, if $u, v, u^{\prime}, v^{\prime} \in K_{r}$ are such that $d(u, v)=d\left(u^{\prime}, v^{\prime}\right)$, then $d(M u, M v)=d\left(M u^{\prime}, M v^{\prime}\right)$. Both $T_{k}$ and $R_{k}$ preserve the oriented distance: this is clear for $T_{k}$ and can easily be checked for $R_{k}^{\prime}$, whence for $R_{k}$. Fix $u=(r, 0)$; explicit computation shows that

$$
u \stackrel{M}{\longmapsto}(1+r: 1-r: 2) \stackrel{R_{k}^{\prime}}{\longmapsto}(1+r:-2 k r-2 r+2: 2) \stackrel{M^{-1}}{\longmapsto}\left(r, \pi / 2 t_{k}(r)\right),
$$

(the second step in the computation uses the matrix $(*)$ displayed above), and therefore $R_{k} M u=\left(R_{k}^{\prime}\right)^{4} M u=M T_{k} u$. We thus obtain $d\left(M T_{k} u, R_{k} M v\right)=$ $d\left(R_{k} M u, R_{k} M v\right)=d(M u, M v)=d\left(M T_{k} u, M T_{k} v\right)$ (because $d(u, v)=$ $\left.d\left(T_{k} u, T_{k} v\right)\right)$. Therefore $R_{k} M v$ and $M T_{k} v$ have the same distance from $M T_{k} u$, whence they are equal.

Until the end of this section $\mu$ is a fixed measure satisfying the hypotheses of Theorem 3.1. The function $\rho:[0,1]^{2} \rightarrow[0,1]$ that maps all points in $Q_{r}$ to $r$ is Borel measurable. Hence the family $\xi=\left\{Q_{r}: r \in[0,1]\right\}$ of its fibers is a measurable decomposition of $[0,1]^{2}$, and $\mu$ disintegrates over $\xi[13$, p. 26]. This means the following: write $\nu=\rho_{*} \mu$ for the push-forward of $\mu$ on $[0,1]$ via $\rho$. Then for every $r$ there exists a Borel probability measure $\mu_{r}$ on $Q_{r}$ such that, for every $\mu$-measurable $A$, we have:

(a) $A \cap Q_{r}$ is $\mu_{r}$-measurable;

(b) the map $r \mapsto \mu_{r}\left(A \cap Q_{r}\right)$ is $\nu$-measurable;

(c)

$$
\mu(A)=\int_{[0,1]} \mu_{r}\left(A \cap Q_{r}\right) d \nu
$$

(d) if $\left\{\mu_{r}^{\prime}\right\}$ is another system of conditional measures satisfying the above properties, then $\mu_{r}^{\prime}=\mu_{r}$ for $\nu$-every $r$.

Lemma 3.3. For $\nu$-every $r$, the conditional measure $\mu_{r}$ coincides with the linear Lebesgue probability measure $\tau_{r}$ on $Q_{r}$.

Proof. It is immediate that $Q_{r}$ contains a rational point iff the intersection point $(1+r: 1-r: 2)$ of $Q_{r}$ with the line segment $\left\langle(1: 1: 2), p_{0}^{0}\right\rangle$ is a rational point iff $r$ is a rational number. Since $\mu$ satisfies $\left(\mathrm{E}^{\prime}\right)$, we have

$$
\nu([0,1] \cap \mathbb{Q})=\mu\left(\bigcup\left\{Q_{r}: r \in[0,1] \cap \mathbb{Q}\right\}\right)=0,
$$

whence we can neglect rational $r$ 's. Fix $k \geq 0$; it suffices to show that the set of all irrational $r$ in the interval $(1 /(2(k+1)+1), 1 /(2 k+1))$ such that $\mu_{r} \neq \tau_{r}$ has $\nu$-measure 0 . Given such an $r$, consider the topological dynamical system $\left(K_{r}, T_{k}\left\lceil K_{r}\right)\right.$ : it is a rotation by an angle of $(1 / r-2 k-1) \pi$, which is an irrational multiple of $\pi$. Irrational rotations are uniquely ergodic, i.e., preserve a unique probability Borel measure [16, $\S 6.5]$. By Lemma 3.2. $\left(K_{r}, T_{k} \uparrow K_{r}\right)$ is topologically conjugate to $\left(Q_{r}, R_{k}\left\lceil Q_{r}\right)\right.$, which is therefore uniquely ergodic as well. Since $R_{k} \uparrow Q_{r}$ preserves the oriented distance, it preserves $\tau_{r}$. On the other hand, by [14, $\S 2.5], R_{k} \uparrow Q_{r}$ preserves $\mu_{r}$ for $\nu$-every $r$; this settles our claim. 
For $i=0, \ldots, 3$, let $T_{i}$ be the open triangle

$$
T_{i}=M\left[\left\{(r, \theta): 0<r<1, i \pi / 2<\theta<(i+1)_{(\bmod 4)} \pi / 2\right\}\right] .
$$

Lemma 3.4. Let $A$ be a Borel subset of the unit square, let $e_{1}=(1,0), e_{2}=(0,1)$ be the standard basis of $\mathbb{R}^{2}$, and let $s \in \mathbb{R}$. If $A, s e_{1}+A \subseteq T_{1} \cup T_{3}$ then $\mu(A)=$ $\mu\left(s e_{1}+A\right)$ and, analogously, if $A, s e_{2}+A \subseteq T_{0} \cup T_{2}$ then $\mu(A)=\mu\left(s e_{2}+A\right)$.

Proof. Since the measures $\mu_{r}$ are linear Lebesgue measures, they are invariant under "horizontal" translations inside $T_{1} \cup T_{3}$, and under "vertical" ones inside $T_{0} \cup T_{2}$. The statement is now immediate from the disintegration property (c).

Lemma 3.5. Let $T \subset \mathbb{R}^{n}$ be a compact convex polyhedron of dimension $n, T^{o}$ its topological interior, $\eta$ a finite Borel measure on $T^{\circ}$. Let us assume that for every $v \in \mathbb{R}^{n}$ and every Borel set $A$ such that $A, v+A \subseteq T^{o}$, we have $\eta(A)=\eta(v+A)$. Then there exists $c>0$ such that $\eta=c \lambda$.

Proof. The proof is modeled on that of [15, Theorem 2.20(d)], so we just sketch it. For $k \geq 0$, a $k$-box is a translate of $\left[0,2^{-k}\right)^{n}$ by a point in $2^{-k} \mathbb{Z}^{n}$. Since the boxes contained in $T^{o}$ generate the Borel $\sigma$-algebra and $\eta, \lambda$ are regular, it suffices to find $c$ such that $\eta(B)=c \lambda(B)$ for every box $B \subset T^{o}$. Let $h$ be minimum such that there exists an $h$-box $C$ in $T^{o}$, and let $c=\eta(C) / \lambda(C)$. Let now $k \geq h$, and let $B$ be any $k$-box in $T^{o}$. Then $C$ is partitioned in $2^{n(k-h)} k$-boxes and each of them, by translation invariance, has the same $\eta$-measure and the same $\lambda$-measure as $B$. Therefore

$$
\eta(B)=\frac{\eta(C)}{2^{n(k-h)}}=\frac{c \lambda(C)}{2^{n(k-h)}}=c \lambda(B) .
$$

In order to apply Lemma 3.5, we need to find another family $\left\{S_{k}: k \geq 0\right\}$ of McNaughton homeomorphisms that force $\mu$ to be invariant under translates along new (i.e., neither horizontal nor vertical) directions. For $k \geq 0$, we consider the following complexes (again, we drawn a picture for $k=1$ ):

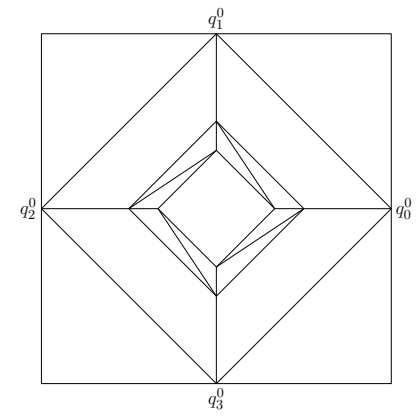

Let $q_{0}^{h}=(h+2: h+1: 2 h+2), q_{1}^{h}=(h+1: h+2: 2 h+2), q_{2}^{h}=(h: h+1: 2 h+2)$, $q_{3}^{h}=(h+1: h+2: 2 h+2)$. The vertices of the exterior rhombus are then $q_{i}^{0}$, for $i=0, \ldots, 3$, those of the intermediate one are $q_{i}^{k}$, and those of the inner one are $q_{i}^{k+1}$. Let $S_{k}^{\prime}$ be the unique homeomorphism that:

(1) $\operatorname{maps} q_{i}^{k+1}$ to $q_{i+1(\bmod 4)}^{k+1}$;

(2) fixes every other vertex;

(3) is affine linear on each polyhedron. 
In this case, $S_{k}^{\prime}$ is not a McNaughton homeomorphism, since the matrices that express the map have rational non-integer entries. Nevertheless, exactly the same proof as in [11, Lemma 3.1] shows that the square of $S_{k}^{\prime}$ is a McNaughton homeomorphism; a fortiori, $S_{k}=\left(S_{k}^{\prime}\right)^{4}$ is a McNaughton homeomorphism.

Define $N: D \rightarrow[0,1]^{2}$ by

$$
N(r, \theta)=(1 / 2,1 / 2)+ \begin{cases}r(\theta / \pi+1 / 2,-\theta / \pi-1), & \text { if }-\pi<\theta \leq-\pi / 2 ; \\ r(\theta / \pi+1 / 2, \theta / \pi), & \text { if }-\pi / 2<\theta \leq 0 ; \\ r(-\theta / \pi+1 / 2, \theta / \pi), & \text { if } 0<\theta \leq \pi / 2 ; \\ r(-\theta / \pi+1 / 2,-\theta / \pi+1), & \text { if } \pi / 2<\theta \leq \pi .\end{cases}
$$

Then $N$ is a homeomorphism between $D$ and the rhombus $E=\left\langle q_{0}^{0}, q_{1}^{0}, q_{2}^{0}, q_{3}^{0}\right\rangle$. Let $V_{r}=N\left[K_{r}\right]$, and define $h_{k}:[0,1] \rightarrow[0,1]$ by

$$
h_{k}(r)=\min (1, \max (0,1 /(2 r)-k-1)) .
$$

Explicit computation, exactly as in the proof of Lemma 3.2, shows that $N$ provides a conjugation between the restriction of $S_{k}$ to $E$ and the twist map $(r, \theta) \mapsto(r, \theta+$ $\left.2 \pi h_{k}(r)\right)$ on $D$. Again, $\mu$ disintegrates along the $V_{r}$ 's, and all the argument leading to Lemma 3.4 carries through. Setting, for $i=0, \ldots, 3$,

$$
H_{i}=N\left[\left\{(r, \theta): 0<r<1, i \pi / 2<\theta<(i+1)_{(\bmod 4)} \pi / 2\right\}\right],
$$

we obtain the following Lemma.

Lemma 3.6. Let $A, e_{1}, e_{2}, s$ be as in the statement of Lemma 3.4 Then:

(i) if $A, s\left(e_{1}+e_{2}\right)+A \subseteq H_{1} \cup H_{3}$ then $\mu(A)=\mu\left(s\left(e_{1}+e_{2}\right)+A\right)$;

(ii) if $A, s\left(-e_{1}+e_{2}\right)+A \subseteq H_{0} \cup H_{2}$ then $\mu(A)=\mu\left(s\left(-e_{1}+e_{2}\right)+A\right)$;

(iii) on $E$, the measure $\mu$ is a positive multiple $c \lambda$ of the Lebesgue measure.

Proof. (i) and (ii) are proved exactly as in Lemma 3.4. By Lemma 3.5, Lemma 3.4 and (i), (ii) above, on each open triangle $T_{i} \cap H_{j}$ the measure $\mu$ is a positive multiple $c_{i j} \lambda$ of Lebesgue. Every $T_{i} \cap H_{j}$ is mapped to any other $T_{i^{\prime}} \cap H_{j^{\prime}}$ by an appropriate symmetry of the square, and these symmetries are McNaughton homeomorphisms. Hence $c_{i j}=c_{i^{\prime} j^{\prime}}$ and (iii) follows since, by Condition $\left(\mathrm{E}^{\prime}\right)$, $\mu\left(E \backslash \bigcup\left\{T_{i} \cap H_{j}: i, j=0, \ldots, 3\right\}\right)=0$.

Each $T_{i} \backslash E$ is the disjoint union of two open triangles, and each of these triangles is the union (modulo $\mu$-null sets, again by $\left(\mathrm{E}^{\prime}\right)$ ) of countably many open rhombi, as in the picture below:

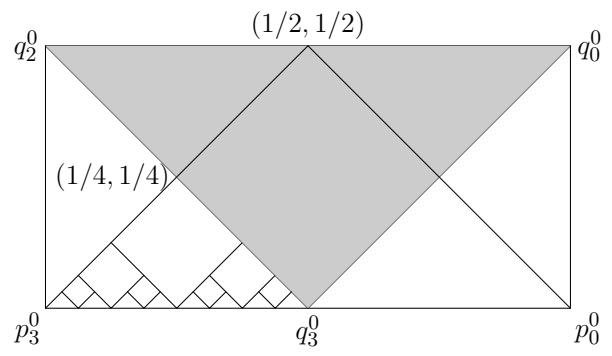

Let $F$ be one of these rhombi, say for definiteness' sake inside the triangle $\left\langle p_{3}^{0}, q_{3}^{0},(1 / 4,1 / 4)\right\rangle$. Let $t$ be the horizontal translation that maps $F$ inside $E$ (the 
grey region above). Then $\mu$ is invariant under translations inside $F$, because any such translation is conjugate via $t$ to a translation inside $E$. By Lemma 3.5. $\mu=c_{F} \lambda$ on $F$, and necessarily $c_{F}$ equals the constant $c$ in Lemma 3.6(iii), because of Lemma 3.4 and the fact that $F, t[F] \subseteq T_{3}$. The union of $E$ with all these countably many rhombi is - modulo $\mu$-null sets - the whole of $[0,1]^{2}$, and therefore $c=1$. This concludes the proof of Theorem 3.1 .

\section{Proof of Theorem 2.3}

Let $\mu^{n}$ be measures as in the statement of Theorem 2.3. If the system $\left\{\mu^{n}\right\}$ is coherent, then $\mu^{n}$ determines $\mu^{m}$ for $1 \leq m \leq n$. By Theorem $3.1 \mu^{2}=\lambda^{2}$, and hence $\mu^{1}=\lambda^{1}$; this settles the last claim in Theorem 2.3. If the system is not coherent, then $\mu^{1}$ may be different from $\lambda^{1}$, as discussed after the statement of Theorem 3.1.

Fix now $n>2$. The natural projection $\pi:[0,1]^{n} \ni\left(x_{1}, \ldots, x_{n}\right) \mapsto\left(x_{3}, \ldots, x_{n}\right) \in$ $[0,1]^{n-2}$ is continuous, whence the set $\xi$ of its fibers is a measurable partition of the $n$-cube. As described after Lemma 3.2. $\mu^{n}$ disintegrates over $\xi$. Let $\nu=\pi_{*} \mu^{n}$ be the push-forward of $\mu^{n}$ on $[0,1]^{n-2}$, and let $\mu_{u}^{n}$ be the conditional measure on $C_{u}=$ $\pi^{-1}\{u\} \in \xi$. The group $G_{2}$ of all McNaughton homeomorphisms of $[0,1]^{2}$ embeds in the group $G_{n}$ of all McNaughton homeomorphisms of $[0,1]^{n}$ in the obvious way: if $R \in G_{2}, p \in[0,1]^{2}$, and $u \in[0,1]^{n-2}$, then we set $R(p, u)=(R(p), u)$.

Lemma 4.1. For every $R \in G_{2}$, every rational polyhedral complex $W$ in $[0,1]^{2}$ such that $|W|$ has empty interior, and $\nu$-every $u \in[0,1]^{n-2}$, the following hold:

(i) $R \uparrow C_{u}$ preserves $\mu_{u}^{n}$;

(ii) $\mu_{u}^{n}\left(\left(|W| \times[0,1]^{n-2}\right) \cap C_{u}\right)=0$.

Proof. By construction, every $C_{u}$ is invariant under the action of $G_{2}$. For a fixed $R \in G_{2}$, the set $U_{R}$ of all $u \in[0,1]^{n-2}$ such that $\mu_{u}$ is invariant under $R \uparrow C_{u}$ has $\nu$ measure 1 . Let $W$ be a complex as stated. Then $|W| \times[0,1]^{n-2}=|V|$ for some rational polyhedral complex $V$ on $[0,1]^{n}$. By condition $\left(\mathrm{E}^{\prime}\right), \mu^{n}(|V|)=0$, and therefore the characteristic function of $|V|$ equals 0 as an element of $L_{1}\left([0,1]^{n}, \mu^{n}\right)$. By the construction of the conditional measures (see, e.g., [12]), the set $U_{W}^{\prime}$ of all $u$ such that $\mu_{u}^{n}\left(|V| \cap C_{u}\right)=0$ has $\nu$ measure 1. Since $G_{2}$ is a countable group and there exist countably many complexes $W$ as above, the set of all $u$ that satisfy both (i) and (ii) has $\nu$ measure 1.

By Lemma 4.1 and Theorem [3.1, for $\nu$-every $u$ the conditional measure $\mu_{u}^{n}$ is the Lebesgue measure $\lambda^{2}$, which is invariant under translations inside $C_{u}$. The disintegration property

$$
\mu^{n}(A)=\int_{[0,1]^{n-2}} \mu_{u}^{n}\left(A \cap C_{u}\right) d \nu
$$

assures then that $\mu^{n}$ is invariant under translations of the form $(p, u) \mapsto(q+p, u)$, for $q \in \mathbb{R}^{2}$. But of course all the above construction can be repeated for any coordinate pair in $[0,1]^{n}$, and therefore $\mu^{n}$ is invariant under any translation inside $[0,1]^{n}$. By Lemma 3.5 $\mu^{n}$ is the Lebesgue measure $\lambda^{n}$.

\section{REFERENCES}

[1] W. M. Beynon. Applications of duality in the theory of finitely generated lattice-ordered abelian groups. Can. J. Math., XXIX(2):243-254, 1977. 
[2] A. Bigard, K. Keimel, and S. Wolfenstein. Groupes et anneaux réticulés, volume 608 of Lecture Notes in Math. Springer, 1977.

[3] R. Cignoli, I. D'Ottaviano, and D. Mundici. Algebraic foundations of many-valued reasoning, volume 7 of Trends in logic. Kluwer, 2000.

[4] A. M. W. Glass. Partially ordered groups, volume 7 of Series in Algebra. World Scientific Publishing Co. Inc., River Edge, NJ, 1999.

[5] K. R. Goodearl. Partially ordered abelian groups with interpolation. American Mathematical Society, Providence, R.I., 1986.

[6] D. Mundici. Interpretation of AF $C^{*}$-algebras in Łukasiewicz sentential calculus. J. of Functional Analysis, 65:15-63, 1986.

[7] D. Mundici. A constructive proof of McNaughton's theorem in infinite-valued logic. J. of Symbolic Logic, 59:596-602, 1994.

[8] D. Mundici. Averaging the truth-value in Eukasiewicz logic. Studia Logica, 55(1):113-127, 1995.

[9] G. Panti. A geometric proof of the completeness of the Lukasiewicz calculus. J. of Symbolic Logic, 60(2):563-578, 1995.

[10] G. Panti. Generic substitutions. J. Symbolic Logic, 70(1):61-83, 2005.

[11] G. Panti. Bernoulli automorphisms of finitely generated free MV-algebras. J. Pure Appl. Algebra, 208(3):941-950, 2007.

[12] M. M. Rao. Conditional measures and applications, volume 177 of Monographs and Textbooks in Pure and Applied Mathematics. Marcel Dekker Inc., New York, 1993.

[13] V. A. Rohlin. On the fundamental ideas of measure theory. Amer. Math. Soc. Transl. Ser., 71:1-54, 1952. Originally published in Mat. Sbornik (N.S.), 25(67):107-150, 1949.

[14] V. A. Rohlin. Selected topics from the metric theory of dynamical systems. Amer. Math. Soc. Transl. Ser. 2, 49:171-240, 1966. Originally published in Uspehi Matem. Nauk (N.S.), $4(2(30)): 57-128,1949$.

[15] W. Rudin. Real and complex analysis. McGraw-Hill Book Co., New York, third edition, 1987.

[16] P. Walters. An introduction to ergodic theory, volume 79 of Graduate Texts in Mathematics. Springer, 1982.

Department of Mathematics, University of Udine, via Delle Scienze 208, 33100 Udine, ITALY

E-mail address: panti@dimi.uniud.it 